\title{
Energy Efficient Routing in Ad Hoc Disaster Recovery Networks
}

\author{
Gil Zussman and Adrian Segall \\ Department of Electrical Engineering \\ Technion - Israel Institute of Technology \\ Haifa 32000, Israel \\ \{gilz@tx, segall@ee\}.technion.ac.il
}

\begin{abstract}
The terrorist attacks on September 11, 2001 have drawn attention to the use of wireless technology in order to locate survivors of structural collapse. We propose to construct an ad hoc network of wireless smart badges in order to acquire information from trapped survivors. We investigate the energy efficient routing problem that arises in such a network and show that since smart badges have very limited power sources and very low data rates, which may be inadequate in an emergency situation, the solution of the routing problem requires new protocols. The problem is formulated as an anycast routing problem in which the objective is to maximize the time until the first battery drains-out. We present iterative algorithms for obtaining the optimal solution of the problem. Then, we derive an upper bound on the network lifetime for specific topologies. Finally, a polynomial algorithm for obtaining the optimal solution in such topologies is described.
\end{abstract}

Keywords-routing, energy efficient, energy conserving, power aware, disaster recovery networks, ad hoc networks, smart badges, IEEE 802.15

\section{INTRODUCTION}

The terrorist attacks on the World Trade Center and the Pentagon on September 11, 2001 have drawn ever-increasing attention to improving rescue efforts following a disaster. One of the technologies that can be effectively deployed during disaster recovery is wireless ad hoc networking [25]. For example, rescue forces can use a Mobile Ad Hoc Network (MANET) in the lack of fixed communication systems. Furthermore, a wireless sensor network can be quickly deployed following a chemical or biological attack in order to identify areas affected by the chemical/biological agents [2]. We propose another application of an ad hoc network, which can be used in order to gather information from trapped survivors of structural collapse.

There are various possible reasons for structural collapse. The most frequent reasons are earthquakes, terror attacks, structural problems, and missile attacks. Regardless of the reason, the consequences of a collapse are usually very tragic. For example, in 1995 alone, the Kobe earthquake resulted in the death of nearly 5,500 people, 168 people were killed in the Oklahoma City bombing, and more than 500 people were killed in the collapse of the Sampoong department store in rael.
Seoul. Thus, the importance of improving rescue techniques requires no explanation.

There are a few techniques for locating survivors of structural collapse trapped in the rubble: fiber optic scopes, sensitive listening devices, seismic sensors, search-and-rescue dogs, etc. [11]. Moreover, during the rescue attempts in the World Trade Center disaster site, the Wireless Emergency Response Team (WERT) attempted to locate survivors through signals from their mobile phones [29].

We propose to extend these capabilities and to enable the location of survivors by acquiring information from their smart badges. Smart badges (a.k.a. RFID badges [30]) will gain increased popularity in the near future and will apparently be used in any modern office building [27]. Since the transmission range of a badge is very short and since rescue equipment can usually be deployed at the periphery of the disaster scene, there is a need to construct an ad hoc network connecting victims trapped in the debris to the rescuers. In such a network, the information acquired from the badges (such as last known location, body temperature, etc.) will be repeatedly routed through other badges to wireless receivers deployed in the disaster scene. The receivers will forward the information through wired or wireless links to a central unit.

In the coming years, smart badges will use a proprietary technology (e.g. [28]) or the new IEEE 802.15.4 standard for Low-Rate Wireless Personal Area Networks (LR-WPAN) [6], [17],[19]. Either way they will be simple devices with very low data rates and very limited power sources. These data rates and power sources are expected to be adequate for regular use. For example, the data rate of an IEEE 802.15.4 device will be 20,40 or $250 \mathrm{~Kb} / \mathrm{s}$ [6]. A smart badge based on this standard is expected to establish about 20 connections per day [27]. Thus, the average data rates are expected to be much lower than the possible data rate. Moreover, the duty cycle of such a device is expected to be less than $1 \%$, thereby enabling a long battery life.

However, in an emergency network constructed after a collapse, which may connect thousands of nodes and may route critical information, the required data rates and the consumed energy may be much higher than in daily use. Thus, the low data rates and the limited power sources are a major constraint on the performance of an emergency network. Furthermore, in 
such a network depleting the battery of a node may have tragic results.

This paper focuses on energy efficient routing protocols for emergency networks of badges. We note that since wireless devices usually have a finite power supply, there is an increasing interest in research regarding energy conserving protocols (see Section 2). Thus, our network model is based on the model for energy conserving routing in a wireless sensor network presented by Chang and Tassiulas [8]. However, unlike a wireless sensor network in which the available bandwidth is usually sufficient, in the emergency network there is a strict bandwidth restriction along with a strict energy restriction. Hence, the solution of the problem calls for the development of new protocols.

We assume that since the proposed network will be composed of trapped survivors' badges, the network topology and the requirements will be rather static. Therefore, our major interest is in distributed algorithms for quasi-static anycast routing in a static network with stationary requirements and unchanging topology. The objective of the algorithms is to maximize the time until the first battery drains-out (i.e. to solve a max-min optimization problem). This objective function has been defined by Chang and Tassiulas [8] and although it is controversial when applied to MANETs or sensor networks, it is appropriate for an emergency network in which every node is critical.

In this paper we formulate the problem and present iterative algorithms for obtaining its optimal solution. These algorithms are based on the formulation of the problem as a concurrent max flow problem [21] and the complexity of one of them is logarithmic in the network lifetime. Then, we derive an upper bound on the network lifetime for specific topologies that is based on the new notion of non-max capacity cut. Finally, a polynomial algorithm for obtaining the optimal solution in specific topologies is described. Due to space constraints, numerical results are not presented in this paper and a few proofs are omitted. The results and the proofs can be found in [39].

The main contribution of this paper is the extension of the energy conserving routing model presented by Chang and Tassiulas [8] to a network in which some of the nodes have a very low data rate as well as a limited battery. Another contribution is the derivation of bounds on the network lifetime and the development of optimal algorithms, which can be implemented in a distributed manner.

This paper is organized as follows. In Section 2, we discuss related work and in Section 3, we present the model and formulate the routing problem. Algorithms for obtaining the solution of the problem and an upper bound on the network lifetime are introduced in Section 4. Section 5 summarizes the main results and discusses future research directions.

\section{RELATED WORK}

In 1998, Bambos [4] reviewed developments in power control in wireless networks and identified the need for mini- mum-power routing protocols. Since then, the issue of energyconservation in ad hoc and sensor networks has attracted a vast amount of research (see for example, [14],[20],[26], and references therein). This research deals with all layers of the protocol stack and is mostly motivated by the fact that wireless devices usually have a very limited power supply. In particular, there is an increasing interest in algorithms for the network layer, namely in energy efficient routing algorithms.

A pioneering work regarding energy efficient routing was presented by Singh et al. [32] who studied via simulation the issue of increasing node and network life by using poweraware metrics for routing. In [36] other power-aware metrics are presented and their performance is studied via simulation.

Some of the previous work regarding energy efficient routing in mobile ad-hoc networks (MANETs) focused on performance comparison of existing ad hoc routing protocols (such as DSR, AODV, TORA, and DSDV [25]) with respect to energy consumption (e.g. [12]). Recently, new power-aware routing protocols for MANETs have been proposed. For example, in [15] a technique (named PARO) designed as a power-aware enhancement for MANET routing protocols has been introduced. In addition, in [38] an algorithm (named GAF) that is designed to reduce the energy consumption in the network by turning off unnecessary nodes and which is independent of the underlying routing protocol is introduced.

We note that Wieselthier et al. have published numerous papers on energy-aware broadcasting and multicasting (see for example, [37] and references therein) and that their work is closely related to the issue of energy efficient routing. For example, Michail and Ephremides [23] study the problem of energy efficient routing of connection oriented traffic. This problem has some relationship to the problem studied in this paper since unlike other authors they take into consideration the fact that the nodes have finite capacity. However, they provide heuristic algorithms whereas we attempt to develop optimal algorithms.

The special characteristics of wireless sensor networks and energy conserving techniques for such networks are described in [2],[33], and in a number of papers related to the MIT's $\mu$ AMPS project [24]. For instance, in [18], an energy efficient routing algorithm based on clustering is described and in [5], a methodology for computing upper bounds on the lifetime of a sensor network is presented.

Chang and Tassiulas [7] introduced one of the first models of energy conserving routing in sensor networks. They defined the energy conserving routing problem as an optimization problem in which the performance objective is to maximize the lifetime of the network (i.e. to maximize the time until the first battery drains-out). They proposed heuristic routing protocols for the solution of the problem and evaluated their performance by simulation.

The work of Chang and Tassiulas [7] has been extended in several different directions. In [8], they have extended their model for the case of multicommodity flow and in [9], they have proposed algorithms for obtaining an approximate solu- 
tion of the routing problem. Moreover, in [22], approximate online algorithms for the case in which the message sequence is not known have been proposed. The problem of finding a flow control strategy that maximizes the sources' utilities subject to a constraint on the network lifetime has been addressed in [34]. In [10], techniques to maximize the network lifetime in the case of cluster-based networks have been devised. A scheme for energy aware routing in a network of piconodes [28] that chooses among possible paths based on a probabilistic fashion has been introduced in [31]. Finally, in this paper, we introduce an extension to the model of Chang and Tassiulas for the case in which the nodes have a very limited bandwidth as well as a limited battery.

\section{FORMULATION OF THE PROBLEM}

\section{A. Model and Preliminaries}

Consider the connected directed network graph $G=(N, L)$. $N$ will denote the collection of nodes $\{1,2, \ldots, n\}$. A node could be a badge, a receiver (the collection of receivers is denoted by $R$ ), or the central unit (referred to as the destination and denoted by $d$ ). Recall that receivers are deployed at the periphery of the disaster scene (their role is to connect the badges network to the central unit).

The collection of the directional links will be denoted by $L$. We assume that since smart badges are intended to be very simple and cheap devices they will usually transmit at a constant power level. Thus, a unit $j$ that is within the transmission range of node $i$ is connected to $i$ by a directional link, denoted by $(i, j)$. For each node $i, Z(i)$ will denote the collection of its neighboring nodes (nodes connected to node $i$ by a directional link).

Let $F_{i j}$ be the average flow on link $(i, j)\left(F_{i j} \geq 0 \quad \forall(i, j) \in L\right)$. We define $f_{i j}$ as the ratio between $F_{i j}$ and the maximal possible flow on a link connecting smart badges ${ }^{1}\left(0 \leq f_{i j} \leq 1\right)$. In the sequel, $f_{i j}$ will be referred to as the flow on link $(i, j)$. The ratio between the rate in which information is generated at badge node $i$ and the maximal possible flow on a link connecting smart badges, is denoted by $r_{i}\left(0 \leq r_{i}<1\right)$.

The transmission energy required by node $i$ to transmit an information unit is denoted by $e_{i}$. Let each node $i$ have an initial energy level $E_{i}$ (we assume that $E_{i}>0 \forall i \in N$ ). If a node $i$ is a receiver or the destination, its energy source is much larger than the energy source of a badge, and therefore, for such a node $E_{i}=\infty$.

For low-power devices operating in the $2.4 \mathrm{GHz}$ ISM band, the transmitter and receiver currents are often similar [17]. Thus, we assume that energy is consumed only when a node transmits information (alternatively, the energy consumed when it receives information can be included in $e_{i}$ ). Moreover, since the energy required in order to receive a message is not negligible, we assume that although a few nodes

${ }^{1}$ For example, in IEEE 802.15.4 the maximal data rate (i.e. the maximal possible flow) is 20,40 or $250 \mathrm{~Kb} / \mathrm{s}$. are able to receive the same message, only the node to which it is intended will receive the full message and forward it. The other nodes will be in sleep mode or communicate with their other neighbors.

We assume that the requirements $\left(r_{i}\right)$ are given. The objective of our energy efficient routing protocols is to obtain link flows $\left(f_{i j}\right)$ such that the time until the first battery drains-out will be maximized. Thus, following the formulation of [8] and using the above assumptions, we define the lifetime of a node and of the network as follows.

Definition 1: (Chang and Tassiulas [8]) The lifetime of node $i$ under a given flow is denoted by $T_{i}$ and is given by:

$$
T_{i}=\frac{E_{i}}{e_{i} \sum_{j \in Z(i)} f_{i j}} .
$$

Definition 2: (Chang and Tassiulas [8]): The lifetime of the network under a given flow is the time until the first battery drains-out, namely the minimum lifetime over all nodes. It is denoted by $T$ and is given by:

$$
T=\min _{i \in N} T_{i}=\min _{i \in N} \frac{E_{i}}{e_{i} \sum_{j \in Z(i)} f_{i j}} .
$$

\section{B. Problem Formulation}

Badges will generate information that will be routed through any of the receivers to the destination. Thus, the resulting problem is an anycast routing problem. Accordingly, the energy efficient routing problem can be formulated as follows.

\section{Problem EER:}

Given: Topology and requirements $\left(r_{i}\right)$

Objective: Maximize the network lifetime:

$$
\max T=\max \left(\min _{i \in N} \frac{E_{i}}{e_{i} \sum_{j \in Z(i)} f_{i j}}\right)
$$

Subject to:

$$
\begin{aligned}
& f_{i j} \geq 0 \quad \forall(i, j) \in L \\
& \sum_{k \in Z(i)} f_{k i}+r_{i}=\sum_{j \in Z(i)} f_{i j} \quad \forall i \in N-\{R, d\} \\
& \sum_{k \in Z(i)} f_{k i}=\sum_{j \in Z(i)} f_{i j} \quad \forall i \in R \\
& \sum_{k \in Z(i)} f_{k i}+\sum_{j \in Z(i)} f_{i j} \leq 1 \quad \forall i \in N-\{R, d\}
\end{aligned}
$$

Constraints (4) - (6) are the usual flow conservation constraints. The meaning of (7) is that the total flow through a node cannot exceed the maximal badge node capacity (i.e. the maximal data rate of a badge).

\section{Numerical Example}

Fig. 1 illustrates a simple network composed of five badges, two receivers, and a single destination. In the optimal 


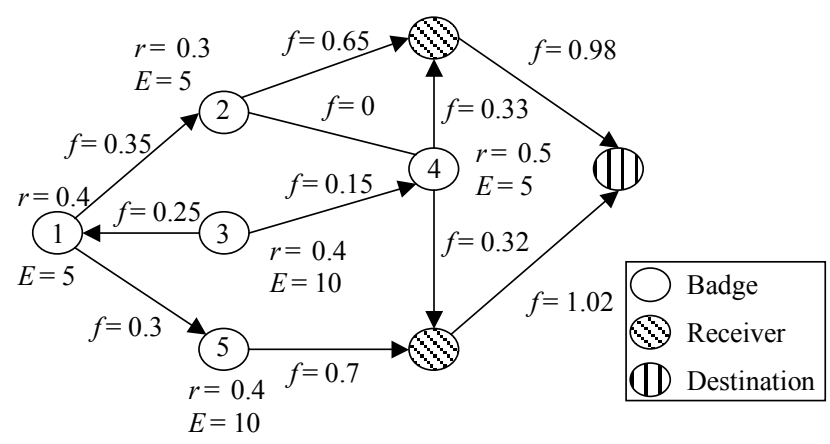

Figure 1. The required transmission rates $\left(r_{i}\right)$, the initial energy values $\left(E_{i}\right)$, and the optimal flows $\left(f_{i j}\right)$ in a network of badges (assuming that $e_{i}=1 \forall i$ )

solution, the network lifetime is 7.69 time units (the batteries of nodes 1,2, and 4 are depleted after 7.69 time units). It can be seen that node 5 , whose battery has remaining power at time 7.69, utilizes its full capacity throughout the operation of the network.

\section{ALGORITHMS AND BOUNDS}

In this section, we present an equivalent formulation of Problem EER. This formulation is required in order to develop distributed algorithms. Then, iterative algorithms for obtaining the optimal solution of the problem are described. We also derive an upper bound on the network lifetime for specific topologies. Finally, we describe a polynomial algorithm for obtaining the optimal solution in these topologies.

\section{A. Linear Programming Formulation}

The first step towards obtaining a solution to Problem EER is converting it to a linear programming problem (Problem EER-LP). Following the approach in [8], we first define $\bar{f}_{i j}$ as the amount of information transmitted from node $i$ to node $j$ until time $T\left(\bar{f}_{i j}=f_{i j} \cdot T\right)$. Then, we formulate Problem EERLP as follows.

\section{Problem EER-LP:}

Given: Topology and requirements $\left(r_{i}\right)$

Objective: Maximize the network lifetime: $\max T$

Subject to: $\quad \bar{f}_{i j} \geq 0 \quad \forall(i, j) \in L$

$\sum_{k \in Z(i)} \bar{f}_{k i}+r_{i} \cdot T=\sum_{j \in Z(i)} \bar{f}_{i j} \quad \forall i \in N-\{R, d\}$

$\sum_{k \in Z(i)} \bar{f}_{k i}=\sum_{j \in Z(i)} \bar{f}_{i j} \quad \forall i \in R$

$e_{i} \sum_{j \in Z(i)} \bar{f}_{i j} \leq E_{i} \quad \forall i \in N-\{R, d\}$

$$
\sum_{k \in Z(i)} \bar{f}_{k i}+\sum_{j \in Z(i)} \bar{f}_{i j} \leq T \quad \forall i \in N-\{R, d\}
$$

Problem EER-LP is a linear programming problem, and therefore, it can be solved by well-known algorithms (e.g. Simplex [1, p. 810]). However, these algorithms cannot be easily modified in order to allow distributed implementation, which is required in an ad hoc network. Thus, analyzing the characteristics of the problem is required in order to develop distributed algorithms.

If the last set of constraints (13) is ignored, Problem EERLP becomes a concurrent max-flow problem with constraints on the flows at the nodes. A concurrent max-flow problem is a multicommodity flow problem in which a demand is associated with each commodity and the objective is to maximize a common fraction of each demand without exceeding the capacity constraints [3],[16],[21]. Accordingly, we define Problem CMF as follows ( $T$ is the common fraction of each demand).

\section{Problem CMF (Concurrent Max Flow):}

Given: Topology and requirements $\left(r_{i}\right)$

Objective: $\max T$ (as in (8))

Subject to: Flow conservation constraints (such as (9)-(11)) Capacity constraints (such as (12))

In the sequel we shall define different instances of Problem $\mathrm{CMF}$ by altering either the flow conservation constraints or the capacity constraints.

Adding (13) to Problem CMF means that $T$ has to be maximized subject to the additional constraint that the flow through a node cannot exceed some percentage of the flow in the network. Recall that (13) results from the fact that the data rate of a badge might be lower than the required bandwidth in an emergency situation. In the following subsections, we shall discuss two different methods for dealing with the complexities imposed by (13).

\section{B. Iterative Algorithms}

An algorithm for obtaining the optimal solution of Problem EER-LP can be based on repeated solutions of different instances of Problem CMF. Following the solution of an instance of Problem CMF, the node capacities, which depend both on the energy (12) and the value of the network lifetime (13), have to be recomputed according to the obtained lifetime $(T)$. Then, another instance of Problem CMF (with the new capacities) has to be solved. This process should be repeated until the optimal solution to Problem EER-LP is obtained.

In this section, we describe an algorithm (referred to as the Iterative Algorithm) based on the above methodology. Then, we present an improved version of the algorithm which utilizes binary search (we shall refer to it as the Binary Iterative Algorithm). We note that both algorithms obtain an optimal solution to Problem EER and that the complexity of the Binary Iterative Algorithm is logarithmic in the network lifetime.

Since there is a single destination node, an instance of Problem CMF can be solved by using binary search with a max flow algorithm (e.g. the preflow-push algorithm [13]). ${ }^{1}$

\footnotetext{
${ }^{1}$ Problem CMF can also be solved by using binary search with a version of the approximate algorithm presented in [3].
} 
Specifically, if for a given set of demands (i.e. for a given $T$ ) there exists a feasible flow (e.g. flow satisfying (8)-(12)), it can be found by a max flow algorithm. Thus, in order to check the feasibility of a given $T$ as a solution to an instance Problem $\mathrm{CMF}$, the network graph should be converted such that every badge node $i$ is connected to a super origin by a link whose capacity is $r_{i} T$. The max flow algorithm should be used in order to maximize the flow from the super origin to the destination. If in the obtained solution the flow outgoing from the super origin is

$$
\sum_{i \in N-\{R, d\}} r_{i} T
$$

then $T$ is feasible.

Accordingly, we define the process of binary search for the solution of Problem CMF as follows.

Definition 3: Algorithm BMF (Binary Max Flow) - A binary search algorithm for the solution of Problem CMF (i.e. for obtaining $T$ ). At each iteration of the binary search (i.e. for a given $T$ ), a max flow algorithm is executed. It is executed in a network with a super origin node which is connected to every badge node $i$ by a link whose capacity is $r_{i} T$.

Since the complexity of a max flow algorithm is $O\left(n^{3}\right)$ [1, p.240],[13], the number of steps required to find a solution to Problem CMF by Algorithm BMF is

$$
O\left(n^{3} \log T_{\max }\right)
$$

where $T_{\max }$ is the maximal possible value of network lifetime $(T)$. It can be shown that for a network of badges and the resulting Problem CMF, the value of $T_{\max }$ is bounded by $n$ times the maximal lifetime of a single node (recall that $n$ is the number of nodes). Consequently, assuming that the required tolerance of the solution is in seconds and that regardless of the traffic, the lifetime of a battery is bounded by about 10 years, $O\left(\log T_{\max }\right)$ is actually $O(\log n)$.

As mentioned before, the solution of Problem EER-LP can be based on solutions of different instances of Problem CMF. Problem EER-LP includes constraints on the node flows. Thus, in order to enable the execution a max flow algorithm (required for the solution of Problem CMF), the network graph should be converted as described in the following. Since the capacities imposed by (12) and (13) are node capacities, each badge node $i$ should be divided into two subnodes $\left(i_{i}\right.$ and $i_{o}$ ) connected by an internal link. If node $i$ generates information, we assume it is generated at $i_{i}$. Accordingly, for a given $T$, the capacity of the internal link $\left(i_{i}, i_{o}\right)$ is defined as:

$$
c_{i_{i} i_{o}}=\min \left(\frac{E_{i}}{e_{i}}, \frac{T\left(1+r_{i}\right)}{2}\right) \quad \forall i \in N-\{R, d\} .
$$

Following the division of the nodes, every directional link $(i, j)$ connecting badge nodes should be replaced by a directional link $\left(i_{o}, j_{i}\right)$. The capacities of all these links should be set to $\infty$. Notice that directional links connecting a badge node $i$ to receiver node $j$ should be replaced by a directional link $\left(i_{o}, j\right)$ with infinite capacity.
In the first iteration of the Iterative Algorithm, the value of $T$ at all the internal links should be set to $\infty$. A solution to the resulting Problem $\mathrm{CMF}$ in the converted network should be obtained by Algorithm BMF. ${ }^{1}$ We shall denote the value of $T$ obtained at this stage by $T_{1}$. Then, the internal link capacities should be updated according to (16) and the value of $T_{1}$. A solution to the resulting Problem CMF in the converted network should be obtained by Algorithm BMF. This process is repeated until the flow values computed by Algorithm BMF satisfy all the internal link capacities (16) computed according to the obtained $T$.

The complexity of the Iterative Algorithm is not necessarily polynomial in the number of nodes or links. Specifically, the number of executions of Algorithm BMF, whose complexity is given in (15), is not necessarily polynomial in the number of nodes or links.

A straightforward improvement is the use of binary search in order to obtain the value of the optimal $T$. Namely, after obtaining $T_{1}$, a max flow algorithm should be used in order to check whether the lifetime of $T_{1} / 2$ is feasible when the internal link capacities are computed according to (16) and $T=T_{1} / 2$. If it is, the feasibility of $3 T_{1} / 4$ should be checked in a similar manner. Otherwise, the feasibility of $T_{1} / 4$ should be checked. The algorithm terminates when the difference between the feasible and non-feasible $T$ is within the required tolerance.

We refer to the algorithm based on the above methodology as the Binary Iterative Algorithm and describe it in Fig. 2. Recall that checking the feasibility of a given network lifetime requires $O\left(n^{3}\right)$ steps. Thus, the complexity of this algorithm is given by (15).

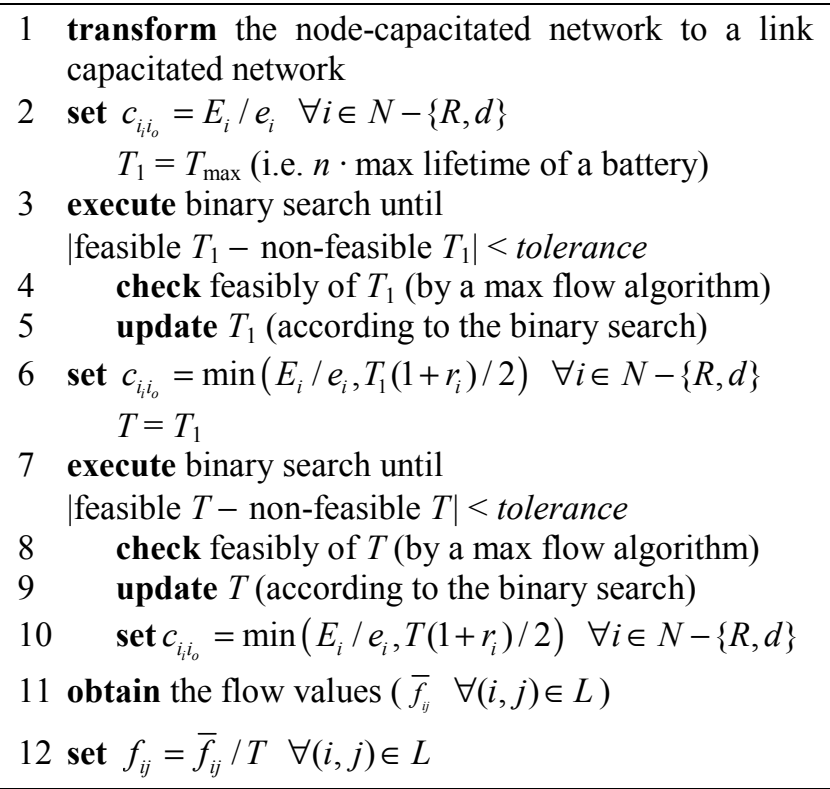

Figure 2. The Binary Iterative Algorithm for obtaining the optimal solution of Problem EER

\footnotetext{
${ }^{1}$ Notice that since $T$ is set to $\infty$ in (16), Problem CMF is equivalent to the problem defined by (8)-(12).
} 
Although max flow algorithms (as the preflow-push algorithm) can be used in environments where decisions have to be made locally, the distributed implementation of the Binary Iterative Algorithm, described in Fig. 2, requires some coordination mechanism. This mechanism is required since the nodes should be aware of the binary search and the value of $T$, which determines the internal link capacities (see steps 6 and 10 in Fig. 2). The definition of the exact procedure in which a distributed iterative algorithm has to be executed is subject for further research.

\section{Upper Bound on the Network Lifetime}

In this section, we derive an upper bound on the network lifetime. It is based on a few observations regarding the relationship between the optimal network lifetime and the capacities of different cuts in the network. The bound can be computed using a max flow algorithm (e.g. the preflow-push algorithm [13]). In the next section, we shall show that in a network with a single origin node, the bound is equal to the optimal solution and outline an $O\left(n^{4}\right)$ algorithm for obtaining the optimal solution.

In this section, we focus on the case in which only a subset of the badges generates information (we shall refer to these badges as the origin nodes and denote the collection of origin nodes by $A$ ). Moreover, we assume that these nodes do not forward information generated by other nodes. ${ }^{1}$ The resulting network graph is described in Fig. 3. In the future, we intend to extend the bound for the case in which all the badges may generate information and forward information of other badges. Moreover, we conjecture that the bound is tight when there is more than a single origin node.

We shall now redefine the transformation of a nodecapacitated network to a link-capacitated network and restate well-known definitions of a cut and related notions [1, p. 177]. In order to incorporate node capacities, the network graph is transformed in a similar manner to the transformation described in the previous section. However, there are two major

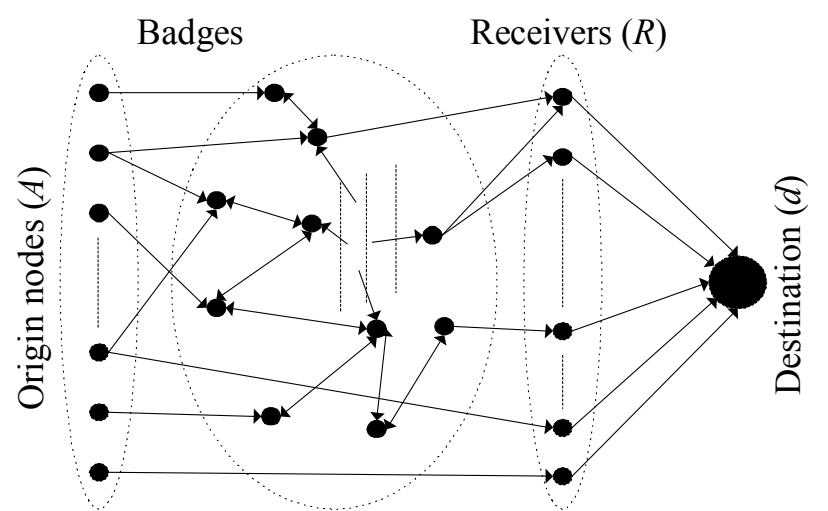

Figure 3. A network graph in which information is generated only by origin nodes. These nodes are not able to forward information generated by other nodes

\footnotetext{
${ }^{1}$ We note that in [35], a similar network topology is studied in the context of routing and scheduling in packet radio networks.
}

differences. First, in some cases, which will be described below, there is no need to separate origin nodes into subnodes. Second, the internal link capacities do not take into account the value of $T$. Accordingly, we define the internal link capacities of badges that are not origins as:

$$
c_{i_{i i_{o}}}=\frac{E_{i}}{e_{i}} \quad \forall i \in N-\{A, R, d\} .
$$

Similarly, in case origin nodes have to be divided into subnodes, their internal link capacities are defined as:

$$
c_{i_{i} i_{o}}=\frac{E_{i}}{e_{i}} \quad \forall i \in A
$$

Notice that according to the context, in the rest of this section, $N$, which originally denotes the collection of nodes, sometimes denotes all the subnodes $\left(i_{i}\right.$ and $\left.i_{o}\right)$.

Definition 4: A cut is identified by a pair $[O, D]$ of complementary subsets of nodes $(D=N-O)$. The capacity of the cut $[O, D]$ is denoted by $C[O, D]$ and is the sum of the capacities of all the links which are directed from $O$ to $D$. The set of links directed from $O$ to $D$ are denoted by $(O, D)$.

We shall now define the new notion of non-max capacity of a cut, which is required in order to determine the upper bound on the network lifetime.

Definition 5: The non-max capacity of the cut $[O, D]$ is the sum of the capacities of the links directed from $O$ to $D$ not including the link with the highest capacity. It is denoted by $Y[O, D]$ and it is given by:

$$
Y[O, D]=C[O, D]-\max _{(i, j) \in(O, D)}\left(c_{i j}\right) .
$$

The next proposition provides an upper bound on the optimal lifetime of the network $\left(T^{*}\right)$.

Proposition 1: If there exists $O \subset N$ in the transformed network with the link capacities determined by (17) that satisfies:

$$
\begin{gathered}
d \notin O \\
\sum_{i \in O} r_{i}>0.5 \\
\forall(i, j) \in(O, D) \text { is an internal link, }
\end{gathered}
$$

then:

$$
T^{*} \leq \min _{\substack{O \subset N: O \text { satisfies } \\(20)-(22)}} \frac{2 Y[O, D]}{2 \sum_{i \in O} r_{i}-1} .
$$

The proof appears in the appendix.

The network lifetime is also bounded by the solution of Problem CMF in the transformed network with the link capacities determined by (17) and (18). It is well known [21] that this solution is bounded by the sparsest cut (a.k.a. min cut), which shall be denoted by $C$ :

$$
C=\min _{O \subset N: d \notin O} \frac{C[O, D]}{\sum_{i \in O} r_{i}} .
$$


We note that in a network with multiple origins and a single destination, the solution to Problem CMF is equal to the sparsest cut (24) [16]. Notice also that the value of $C$ is computed according to the transformed network determined by (17) and (18), whereas the bound described in Proposition 1 is computed according to the transformed network determined only by (17).

The next theorem combines the results of Proposition 1 and (24). The proof can be found in [39]. It is mostly based on Proposition 1 and Lemma 2 which appears in the appendix.

\section{Theorem 1:}

$$
T^{*}\left\{\begin{array}{ccc}
= & C & \sum_{i \in N} r_{i} \leq 0.5 \\
\leq \min \left(\min _{\substack{O \subset N: O \text { satisfies } \\
(20)-(22)}} \frac{2 Y[O, D]}{2 \sum_{i \in O} r_{i}-1}, C\right) & \sum_{i \in N} r_{i}>0.5
\end{array}\right.
$$

As mentioned before, the value of $C$ is the solution to Problem CMF, and therefore, it can be computed by Algorithm BMF, defined in Definition 3. We shall now show that in addition, the computation of the bound on $T^{*}$, described in Theorem 1, requires several iterations of a max flow algorithm. Furthermore, Corollary 1 will show that in a network with a single origin node, the optimal network lifetime $\left(T^{*}\right)$ can be computed by $O(n)$ iterations of a max flow algorithm.

Consider a subgroup of origin nodes (denoted without loss of generality by $\left.\left\{i_{1}, \ldots, i_{k}\right\}\right)$ such that $\left\{i_{1}, \ldots, i_{k}\right\} \in A$ and

$$
\sum_{i \in\left\{i_{1}, \ldots, i_{k}\right\}} r_{i}>0.5 \text {. }
$$

For such a group there may be a few possible cuts $[O, D]$ such that $\left\{i_{1}, \ldots, i_{k}\right\} \in O$ and $\left\{d, A-\left\{i_{1}, \ldots, i_{k}\right\}\right\} \in D$. Since the sum of $r_{i}$ is equal for all these cuts, obtaining the bound described in Proposition 1 (23), is equivalent to obtaining

$$
Y_{\min }\left\{i_{1}, \ldots, i_{k}\right\}=\min _{\substack{O \subset N: O \text { satisfies }(22) \\\left\{i_{1}, \ldots, i_{k}\right\} \in O,\left\{d, A-\left\{i_{1}, \ldots, i_{k}\right\}\right\} \in D}} Y[O, D]
$$

for every subgroup $\left\{i_{1}, \ldots, i_{k}\right\}$. Namely, for every subgroup of origin nodes satisfying (26), the minimum of the non-max capacities of the cuts that separate $\left\{i_{1}, \ldots, i_{k}\right\}$ and $\left\{d, A-\left\{i_{1}, \ldots, i_{k}\right\}\right\}$, and which are composed of internal links should be computed.

We shall now define the notion of an internal-zero Graph, which is required in order to compute $Y_{\min }\left\{i_{1}, \ldots, i_{\mathrm{k}}\right\}$ (27).

Definition 6: The internal-zero Graph $G_{i}$ ( $i \in N-\{A, R, d\}$ ) is identical to the Graph $G$ except that the capacity $c_{i_{i} i_{o}}$ of the internal link $\left(i_{i}, i_{o}\right)$ is taken to be 0 .

According to the following proposition, $Y_{\min }\left\{i_{1}, \ldots, i_{\mathrm{k}}\right\}$ is equal to the minimum of the values of minimal capacity of an $[\mathrm{O}, \mathrm{D}]$ cut (min $C[O, D])$ separating $\left\{i_{1}, \ldots, i_{k}\right\}$ and $\left\{d, A-\left\{i_{1}, \ldots, i_{k}\right\}\right\}$ in $O(n)$ different internal-zero Graphs $\left(G_{i}\right)$. The proof of the proposition is by contradiction and it can be found in [39].

\section{Proposition 2:}

$$
Y_{\min }\left\{i_{1}, \ldots, i_{k}\right\}=\min _{G_{i}: \forall i \in N-\{A, R, d\}}\left(\min _{\substack{O \subset N:\left\{i_{1}, \ldots, i_{k}\right\} \in O,\left\{d, A-\left\{i_{1}, \ldots, i_{k}\right\}\right\} \in D}} C[O, D]\right)
$$

Consequently, for every subgroup $\left\{i_{1}, \ldots, i_{k}\right\} \in A$ satisfying (26), there is a need to obtain the capacity of the minimum cut in $O(n)$ different graphs ${ }^{1}$. According to the Max-Flow MinCut Theorem [1, p. 185], for each of the $O(n)$ graphs the solution of a max flow problem is equal to the min cut. The max flow problem should be solved when the subgroup of origin nodes is connected to a super origin and the objective is to maximize the flow from super origin to the destination.

To conclude, the bound defined in Theorem 1 can be computed by Algorithm BMF and by $O(n)$ executions of a max flow algorithm (e.g. the preflow-push algorithm) for every subgroup of origin nodes satisfying (26). Although the complexity of a max flow algorithm is $\mathrm{O}\left(n^{3}\right)$ [1, p.240],[13], the computation of the bound becomes impractical for very large sets of origins. However, in the next section we use the methodology described above for developing an $O\left(n^{4}\right)$ optimal algorithm for a network with a single origin node.

\section{Non-max Capacity Algorithm}

We have mentioned that the complexity of the Binary Iterative Algorithm, described in Section 4-B, is not necessarily polynomial in the number of nodes or links. Thus, we have developed an optimal energy efficient routing algorithm with polynomial complexity. The algorithm is based on the upper bound derived in Theorem 1 and on the notion of non-max capacity, defined in Definition 5. Therefore, it is referred to as the Non-max Capacity Algorithm.

In the following theorem we shall show that in a network with a single origin node (i.e. only a single badge generates information), the upper bound described in Theorem 1 is equal to the optimal solution. This observation will be used in order to develop an $O\left(n^{4}\right)$ algorithm for obtaining the optimal flow values. We emphasize that a complexity of $O\left(n^{4}\right)$ is usually much lower than the complexity of executing a linear programming algorithm, such as the Simplex.

Theorem 2: If $\left|\left\{i \mid i \in N, r_{i} \neq 0\right\}\right|=1$, the optimal network lifetime $\left(T^{*}\right)$ is equal to the upper bound defined in (25).

The proof appears in the appendix.

Accordingly, it is obvious that if $r_{i} \leq 0.5$ (where $i$ is the origin node), the optimal solution to Problem EER-LP is the sparsest cut ( $C$ defined in (24)). Since there is only a single origin, the value of $C$ can be obtained by a max flow algorithm. Thus, in this case the Non-max Capacity Algorithm reduces to a single execution of the max flow algorithm in the transformed network with the link capacities determined by (17) and (18).

On the other hand, if $r_{i}>0.5$, the Non-max Capacity Algorithm consists of $O(n)$ executions of the max flow algorithm in

\footnotetext{
${ }^{1}$ The graphs are actually the same. The only difference is in the link capacities.
} 
order to obtain the optimal solution to Problem EER-LP. According to Theorems 1 and 2, obtaining the optimal lifetime $\left(T^{*}\right)$ requires computing the values of the sparsest cut $(C)$ and the value of $Y_{\min }\{i\}$ (defined in (27)). We have already mentioned that the value of $C$ can be obtained using a max flow algorithm. Moreover, according to Proposition 2, $Y_{\min }\{i\}$ can be obtained by $O(n)$ executions of a max flow algorithm in the transformed network with the link capacities determined by (17). In each of these executions, $c_{i_{i} i_{o}}=0$ for a different internal link of a badge node.

Once the optimal network lifetime $\left(T^{*}\right)$ is obtained, the internal capacities of the nodes should be updated:

$$
\begin{gathered}
c_{i_{i i_{o}}}=\min \left(\frac{E_{i}}{e_{i}}, \frac{T^{*}}{2}\right) \quad \forall i \in N-\{A, R, d\} \\
c_{i_{i i_{o}}}=\frac{E_{i}}{e_{i}} \quad i \in A
\end{gathered}
$$

Then, a max flow algorithm should be executed in the resulting transformed network in order to derive the flow values $\left(\bar{f}_{i j}\right)$. In the course of the proof of Theorem 2, we have shown that the flow values derived in this procedure yield the optimal network lifetime $\left(T^{*}\right)$. The optimal flow values $\left(f_{i j}\right)$, corresponding to the original problem (Problem EER), can be easily derived from the values of $\bar{f}_{i j}$.

The Non-max Capacity Algorithm, which is based on the above methodology, is described in Fig. 4. It can be seen that it requires $O(n)$ executions of a max flow algorithm. Hence, the following corollary results from the fact that the complexity of a max flow algorithm is $O\left(n^{3}\right)$.

Corollary 1: If $\left|\left\{i \mid i \in N, r_{i} \neq 0\right\}\right|=1$, the value of the network lifetime can be computed by an $O\left(n^{4}\right)$ algorithm.

The Non-max Capacity Algorithm requires $O(n)$ executions of a max flow algorithm such as the preflow-push algorithm [13]. As described in steps 6-9 in Fig. 4, most of these executions can be performed in parallel. After the value of $T^{*}$ is obtained, there is a need to execute a max flow algorithm once more (step 11 in Fig. 4). Since the preflow-push algorithm can be executed in a distributed manner and since the Non-max Capacity Algorithm requires running $O(n)$ instances of the preflow-push algorithm in parallel, the Non-max Capacity Algorithm can be implemented as a distributed algorithm.

Finally, we note that the scalability of the Non-max Capacity Algorithm to a network with multiple origin nodes requires further research.

\section{CONCLUSIONS AND FUTURE RESEARCH}

We have proposed to enable the formation of a network composed of smart badges in order to acquire information from survivors of structural collapse. The two main aspects that affect the performance of such a network are the limited batteries of the badges and their very low data rates (relatively to the requirements in a disaster scene).
1 transform the node-capacitated network to a link capacitated network

2 set $c_{i_{i} i_{o}}=E_{i} / e_{i} \quad \forall i \in N-\{R, d\}$

3 obtain the max flow values $\left(\bar{f}_{i j} \forall(i, j) \in L\right)$

4 if $r_{k}>0.5 k \in A$ ( $k$ is the origin node)

5 set $T_{k}=\bar{f}_{k_{i} k_{o}} / r_{k}$

$6 \quad \forall l \in N-\{A, R, d\}$

$7 \quad$ set $c_{l_{i} l_{o}}=0$

8 obtain the max flow value $\left(\bar{f}_{k_{i} k_{o}}\right)$

$9 \quad$ set $T_{i}=2 \bar{f}_{k_{i} k_{o}} /\left(2 r_{k}-1\right)$

$$
c_{l_{i} l_{o}}=E_{l} / e_{l}
$$

10

$$
\text { set } \begin{aligned}
T^{*} & =\min _{i \in N-\{R, d\}} T_{i} \\
c_{i_{i} i_{o}} & =\min \left(E_{i} / e_{i}, T^{*} / 2\right) \quad \forall i \in N-\{A, R, d\} \\
c_{k_{i} k_{o}} & =E_{k} / e_{k}
\end{aligned}
$$

11 obtain the max flow values $\left(\bar{f}_{i j} \forall(i, j) \in L\right)$

12 set $f_{i j}=\bar{f}_{i j} \cdot r_{k} / \bar{f}_{k_{i} k_{o}} \forall(i, j) \in L$

Figure 4. The Non-max Capacity Algorithm for obtaining the optimal solution of Problem EER in a network with a single origin node

Accordingly, an energy efficient routing problem in such a network has been formulated as an anycast routing problem. The problem has been formulated such that the objective function is to maximize the time until the first battery drains-out and the flow through the badges is bounded by their data rates.

We have presented iterative algorithms for obtaining the optimal solution of the problem. These algorithms are based on the formulation of the problem as a concurrent max flow problem and the complexity of one of them is logarithmic in the network lifetime. Then, we have derived an upper bound on the network lifetime for specific topologies. The bound is based on the new notion of non-max capacity of a cut and is the basis for optimal algorithms. Finally, an $O\left(n^{4}\right)$ distributed algorithm for obtaining the optimal solution in a network with a single origin node has been described. Several numerical examples can be found in [39].

The work presented here is the first approach towards an analysis of the routing problem in an emergency network of smart badges. Hence, there are still many open problems to deal with. For example, we would like to investigate the tightness of the upper bound, derived in this paper, and to evaluate the scalability of the Non-max Capacity Algorithm to networks with several origin nodes. We also wish to utilize methods developed for fractional packing problems in order to develop fast approximate algorithms. In addition, future study will focus on the distributed implementation of the proposed algorithms. For instance, we wish to study how much energy and how many messages are required in order to obtain the optimal solution.

Finally, we note that despite the theoretical importance of the optimal algorithms and bounds, in an emergency situation 
there is a need for low complexity heuristic algorithms. Thus, a major future research direction is the development of approximate and heuristic algorithms that will deal with the special characteristics of a smart badges network operated in a disaster site.

\section{ACKNOWLEDGMENT}

We would like to thank Dr. Oktay Gunluk for insightful comments. We would like to thank the anonymous reviewers for their helpful comments.

\section{APPENDIX}

In this appendix, we outline the proofs of some of the results presented in the paper. The full details appear in [39].

The following lemma is required for the proof of Proposition 1.

Lemma 1: Assume that there exists a feasible flow in the network (i.e. a flow $\bar{f}_{i j}$ satisfying (9)-(13)). Every cut $[O, D]$ in the transformed network (with the link capacities determined by (17)) that satisfies (20)-(22) must include at least 2 internal links.

Proof: According to the Max-Flow Min-Cut Theorem $[1$, p. 185], the flow through the cut $[O, D]$ is at least

$$
T \sum_{i \in O} r_{i}
$$

Thus, according to (21), the flow through the cut is bigger than $T / 2$. Assume that the cut includes only a single internal link $\left(i_{\mathrm{i}}, i_{\mathrm{o}}\right)$. Then, in the original network:

$$
\sum_{k \in Z(i)} \bar{f}_{k i}+\sum_{j \in Z(i)} \bar{f}_{i j}>T
$$

Equation (32) does not satisfy (13) and contradicts the assumption that there exists a feasible flow in the network.

Proof of Proposition 1: We assume that there exists a feasible flow in the network and consider the transformed network (with the link capacities determined by (17)). According to Lemma 1 , every cut $[O, D]$ satisfying (20)-(22) includes at least 2 internal links.

For a cut $[O, D]$, the flow through the link with the highest capacity cannot exceed $T / 2$. Namely:

$$
\bar{f}_{m_{i} m_{o}} \leq \frac{T}{2}=\frac{\sum_{i \in O \cap A} \sum_{k \in Z(i)} \bar{f}_{i k}}{2 \sum_{i \in O} r_{i}}
$$

where $\left(m_{i}, m_{o}\right)$ is the internal link with the maximum capacity in the cut. Thus, the flow through the rest of the links in the cut satisfies:

$$
\sum_{\substack{\left(l_{i}, l_{o}\right) \in(O, D),\left(l_{i}, o_{o} \neq\left(m_{i}, m_{o}\right)\right.}} \bar{f}_{l_{i} l_{o}} \geq \frac{\left(2 \sum_{i \in O} r_{i}-1\right) \sum_{i \in O \cap A} \sum_{k \in Z(i)} \bar{f}_{i k}}{2 \sum_{i \in O} r_{i}}=\frac{\left(2 \sum_{i \in O} r_{i}-1\right) T}{2}
$$

Hence, (23) results from the fact that the non-max capacity of a cut is at least the flow through the links composing the cut, not including the flow through the link with the highest capacity. Namely for every cut $[\mathrm{O}, \mathrm{D}]$ :

$$
Y[O, D] \geq \sum_{\substack{\left(l_{i}, l_{o}\right) \in(O, D),\left(l_{i}, l_{o}\right) \neq\left(m_{i}, m_{o}\right)}} \bar{f}_{l_{i} l_{o}}
$$
1.

The following lemma is required for the proof of Theorem

$$
\text { Lemma 2: } T^{*}=C(C \text { defined in (24) }), \text { if }
$$

$$
\sum_{i \in N} r_{i} \leq 0.5 \text {. }
$$

Proof: Assuming that the flow is loop free, if (36) holds, the maximal possible flow through an internal link cannot exceed $T$. Thus, (13) becomes redundant and Problem EERLP reduces to Problem CMF, whose optimal solution is $C$.

The proof of Theorem 2 is based on the following lemma. Proving the lemma requires considering various cases regarding the capacities of cuts composed of internal links. In particular, the relationships between the capacity of the highest capacity link in a cut and the capacities of the rest of the links as well as the capacities of other cuts are considered. The proofs are omitted and can be found in [39].

Lemma 3: If $\left|\left\{i \mid i \in N, r_{i} \neq 0\right\}\right|=1$, (36) does not hold, and

$$
C<\min _{\substack{O \subset N: O \text { satisfies } \\(20)-(22)}} \frac{2 Y[O, D]}{2 \sum r_{i}-1},
$$

then $T^{*}=C$.

Lemma 4: If $\left|\left\{i \mid i \in N, r_{i} \neq 0\right\}\right|=1$, (36) does not hold, and (37) does not hold, then:

$$
T^{*}=\min _{\substack{O \subset N: O \text { satisfies } \\(20)-(22)}} \frac{2 Y[O, D]}{2 \sum_{i \in O} r_{i}-1} .
$$

Proof of Theorem 2: If (36) holds the theorem is derived from Lemma 2. Consider the case that (36) does not holds and there is a direct link from the origin to a receiver. The origin can transmit directly to the receiver and (13) becomes redundant. Thus, Problem EER-LP reduces to Problem CMF, whose optimal solution is $C$. Notice that in this case the value defined in (38) does not exist since there is no cut $[O, D]$ that satisfies (20)-(22).

If (36) does not hold and there is no direct link from the origin to a receiver, $T^{*}$ is derived from the results of lemmas 3 and 4.

\section{REFERENCES}

[1] R. K. Ahuja, T. L. Magnanti, and J. B. Orlin, Network Flows, Prentice Hall, 1993.

[2] I. F. Akyildiz, W. Su, Y. Sankarasubramaniam, and E. Cayirci, "Wireless Sensor Networks: a Survey”, Computer Networks, Vol. 38, pp. 393422, Mar. 2002. 
[3] B. Awerbuch and T. Leighton, "Improved Approximation Algorithms for the Multi-Commodity Flow Problem and Local Competitive Routing in Dynamic Networks", Proc. ACM STOC'94, May 1994.

[4] N. Bambos, "Toward Power-Sensitive Network Architectures in Wireless Communications: Concepts, Issues, and Design Aspects", IEEE Personal Comm., Vol. 5, pp. 50-59, June 1998.

[5] M. Bhardwaj, T. Garnett, and A. P. Chandrakasan, "Upper Bounds on the Lifetime of Sensor Networks", Proc. ICC'01, June 2001.

[6] E. Callaway, P. Gorday, L. Hester, J. A. Gutierrez, M. Naeve, B. Heile, and V. Bahl, "Home Networking with IEEE 802.15.4: A Developing Standard for Low-Rate Wireless Personal Area Networks", IEEE Comm., Vol. 40, pp. 70-77, Aug. 2002.

[7] J. H. Chang and L. Tassiulas, "Routing for Maximum System Lifetime in Wireless Ad-hoc Networks", Proc. 37th Annual Allerton Conf. on Comm., Control, and Comp., Sep. 1999.

[8] J. H. Chang and L. Tassiulas, "Energy Conserving Routing in Wireless Ad-hoc Networks", Proc. IEEE INFOCOM'00, Mar. 2000.

[9] J. H. Chang and L. Tassiulas, "Fast Approximate Algorithms for Maximum Lifetime Routing in Wireless Ad-hoc Networks", Proc. IFIP-TC6 Networking 2000, LNCS, Vol. 1815, Springer, May 2000.

[10] C. F. Chiasserini, I. Chlamtac, P. Monti, and A. Nucci, "Energy Efficient Design of Wireless Ad Hoc Networks", Proc. IFIP-TC6 Networking 2002, LNCS, Vol. 2345, Springer, May 2002.

[11] Federal Emergency Management Agency (FEMA) - The National Urban Search and Rescue Response System, Documentation available at URL http://www.fema.gov/usr, Dec. 2002.

[12] L. M. Feeney, “An Energy Consumption Model for Performance Analysis of Routing Protocols for Mobile Ad Hoc Networks", Mobile Networks and Applications, Vol. 6, pp. 239-249, June 2001.

[13] A. V. Goldberg and R. E. Tarjan, "A New Approach to the Maximum Flow Problem", J. of the ACM, Vol. 35, pp. 921-940, Oct. 1988.

[14] A. Goldsmith and S. B. Wicker (eds.), "Special Issue: Energy-Aware Ad Hoc Wireless Networks", IEEE Wireless comm., Vol. 9, Aug. 2002.

[15] J. Gomez, A.T. Campbell, M. Naghshineh, and C. Bisdikian, "Conserving Transmission Power in Wireless Ad Hoc Networks", Proc. ICNP'01, Nov. 2001.

[16] O. Gunluk, "A New Min-cut Max-flow Ratio for Multicommodity Flows", Proc. IPCO 2002, LNCS, Vol. 2337, Springer, May 2002.

[17] J. A. Gutierrez, M. Naeve, E. Callaway, M. Bourgeois, V. Mitter, and B. Heile, "IEEE 802.15.4: A Developing Standard for Low-Power LowCost Wireless Personal Area Networks", IEEE Network, Vol. 15, pp. 1219, Sep./Oct. 2001.

[18] W. R. Heinzelman, A. Chandrakasan, and H. Balakrishnan, "EnergyEfficient Communication Protocol for Wireless Microsensor Networks", Proc. HICSS-33, Jan 2000.

[19] IEEE 802.15.4 Standard, Documentation available at URL http://www.ieee802.org/15/pub/TG4.html, Dec. 2002.

[20] C. E. Jones, K. M. Sivalingam, P. Agrawal, and J. C. Chen, "A Survey of Energy Efficient Network Protocols for Wireless Networks", Wireless Networks, Vol. 7, pp. 343-358, July 2001.
[21] T. Leighton and S. Rao, "Multicommodity Max-Flow Min-Cut Theorems and Their Use in Designing Approximation Algorithms", J. of the $A C M$, Vol. 46, pp. 782-832, Nov. 1999.

[22] Q. Li, J. Aslam, and D. Rus, "Online Power-aware Routing in Ad-hoc Networks”, Proc. ACM MOBICOM'01, July 2001.

[23] A. Michail and A. Ephremides, "Energy Efficient Routing for Connection-Oriented Traffic in Ad-Hoc Wireless Networks", Proc. IEEE PIMRC'00, Sep. 2000.

[24] The MIT $\mu$ AMPS Project, Documentation available at URL http://www-mtl.mit.edu/research/icsystems/uamps, Dec. 2002.

[25] C. E. Perkins, Ad Hoc Networking, Addison-Wesley, 2001.

[26] C. Petrioli, R. R. Rao, and J. Redi (eds.), "Special Issue: Energy Conserving Protocols", Mobile Networks and Applications, Vol. 6, June 2001.

[27] D. Pokrajac, "Smart Badge Usage Model", doc. no. IEEE802.1501/039r0, Submitted Jan. 2001.

[28] J. M. Rabaey, M. J. Ammer, J. L. daSilva Jr., D. Patel, and S. Roundy, "PicoRadio Supprorts AD Hoc Ultra-Low Power Wireless Networking", IEEE Computer, Vol. 33, pp. 42-48, July 2000.

[29] K. F. Rauscher, "Wireless Emergency Response Team - Final Report for the Sep. 112001 NYC WTC Terrorist Attack", Oct. 2001.

[30] The RFID page (AIM-AIDC), Documentation available at URL http://www.aimglobal.org/technologies/rfid, Dec. 2002.

[31] R. C. Shah and J. M. Rabaey, "Energy Aware routing for Low Energy Ad Hoc Sensor Networks", Proc. IEEE WCNC'02, Mar. 2002.

[32] S. Singh, M. Woo, and C.S. Raghavendra, "Power-Aware Routing in Mobile Ad Hoc Networks", Proc. ACM/IEEE MOBICOM'98, Oct. 1998

[33] K. Sohrabi, J. Gao, V. Ailawadhi, and G. J. Pottie, "Protocols for SelfOrganization of a Wireless Sensor Network", IEEE Personal Comm., Vol. 7, pp. 16-27, Oct. 2000.

[34] V. Srinivasan, C. F. Chiasserini, P. Nuggehalli, and R. Rao, “Optimal Rate Allocation and Traffic Splits for Energy Efficient Routing in Ad Hoc Networks", Proc. IEEE INFOCOM'02, June 2002.

[35] L. Tassiulas and A. Ephremides, "Jointly Optimal Routing and Scheduling in Packet Radio Networks", IEEE Tran. on Information Theory, Vol. 38, pp. 165-168, Jan. 1992.

[36] C. K. Toh, "Maximum Battery Life Routing to Support Ubiquitous Mobile Computing in Wireless Ad Hoc Networks", IEEE Comm., Vol. 39, pp. 138-147, June 2001.

[37] J. E. Wieselthier, G. D. Nguyen, and A. Ephremides, "Resource Management in Energy-Limited, Bandwidth-Limited, Transceiver-Limited Wireless Networks for Session-Based Multicasting", Computer Networks, Vol. 39, pp. 113-131, June 2002.

[38] Y. Xu, J. Heidemann, and D. Estrin, "Geography-informed Energy Conservation for Ad-hoc Routing”, Proc. ACM MOBICOM'01, July 2001.

[39] G. Zussman and A. Segall, "Energy Efficient Routing in Ad Hoc Disaster Recovery Networks", CCIT Report 392, Technion - Dept. of Electrical Engineering, July 2002. Available at URL http://www.comnet.technion.ac.il/segall/reports/ccit_392.pdf 